\title{
Prevalent Non-communicable Diseases Across Brazil: Risk Factors, Prevention, and Future Goals
}

\author{
5. A. Borgia (Sarah A. Borgia)
}

Health Administration \& Human Resources,

Original Articles

$4^{\text {th }}$ Floor McGurrin Hall, Scranton, PA 18510-4699

\section{E-mail address:}

sarah.borgia@scranton.edu

\section{Reprint address:}

Health Administration \&

Human Resources

$4^{\text {th }}$ Floor McGurrin Hall

Scranton, PA 18510-4699

Suource: Clinical Social Work and Health Intervention

Volume: 7

Issue: 4

Pages: $62-66$

Cited references: 0

\section{Reviewers:}

Prof. Dr. Roberto Cauda, Ph.D.

Institute of Infectious Diseases, Catholic University of the Sacred Heart, Rome, IT

e-mail: roberto.cauda@Unicatt.it

Dr. Zoltán Pataky

HÚG v Ženeve, CH

e-mail: zoltan.pataky@hcuge.ch

\section{Key words:}

Non-communicable diseases, Risk factors, Prevention.

\section{Publisher:}

International Society of Applied Preventive Medicine i-gap

CSWHI 2016; 7(4): 62 - 66; DOI 10.22359/cswhi_7_4_09 @ 2016 Clinical Social Work and Health Intervention

\section{Abstract:}

Over the past century, the country of Brazil has seen tremendous growth in their economy, including - increased mechanization, urbanization, and industrialization. Collectively, these changes have made a negative impact in the public health sector in Brazil because they have contributed to the increasing number of individuals who have developed a non-communicable disease. Non-communicable diseases (NCDs), also known as chronic diseases, are developed over time and are caused 
by a various number of risk factors. With these risk factors uncontrolled, the burden only continues to rise and have a profound impact on the health and quality of life for any individual living with a NCD. Additionally, those individuals living with a NCD can be heavily burdened financially, psychically, and socially. Currently, NCD's represent the largest mortality and morbidity burden in the Brazil. The increasing burden of NCD's have not only become a serious public health issue for the country of Brazil, but for many countries across the globe. An important way to reduce NCDs is to focus on lessening the risk factors associated with these diseases. Currently, Brazil has implemented and continues to develop solutions to reduce this burden but they still have a long way to go. This paper will examine the impact of NCD's on the population of Brazil including: risk factors associated with these chronic diseases; how this country' prevalence of NCD's compares to other countries across the globe; what is being done throughout Brazil to combat this growing burden.

\section{Introduction}

Having good health is something to be thankful for because it is not something that everyone possesses due to various ailments such as non-communicable diseases (NCD). Living with an NCD can cause a significant burden on not just one's health, but one's overall quality of life. There are several elements about NCDs which can provide clearer understanding of what they are.

First, NCD cannot be spread from person to person by an infectious agent, even if they might be associated with one. Second, they tend to last a long time. Third, they can be very disabling, can seriously impair the ability of people to engage in day-to-day activities, and often lead to death if they are not treated appropriately. NCDs kill 38 million people each year and almost three quarters of NCD deaths - 28 million - occur in low and middle income countries. The four main types of Non-communicable Diseases are cardiovascular diseases (like heart attacks and stroke); cancer; chronic respiratory diseases (such as chronic obstructed pulmonary disease and asthma); diabetes. In Brazil, cardiovascular diseases accounted for the highest percentage of NCDs, followed by cancer, other NCDS such as respiratory diseases, and finally diabetes, as shown in Table 1. Additionally, the main risk factors that contribute to the development of NCD's include tobacco use, physical inactivity, unhealthy diet and the harmful use of alcohol. Strategies that focus on lessening the risk factors that lead to NCDs is a vital element in the aim to reduce the large burdens they create for Brazil and so many other countries worldwide.

\section{Impact in Brazil}

Brazil, a country that is filled with beautiful beaches, mountains and home to many famous tourist attractions to which individuals travel from all over the world travel to see, faces the unwavering burden of non-communicable diseases (NCD's). There are several statistics that can provide a clearer idea of how negatively this burden impacts Brazil. For example, in $2007,72 \%$ of all deaths were attributable to NCD's. Additionally, they are the main source of disease burden in Brazil. Next, the percentage of avoidable chronic disease hospitalizations in Brazil was 20\% in 2007, for adults ages 20-79. Lastly, the economic impact of NCDs in Brazil was $\$ 72$ million in 2005. (World Bank 2005) The driving forces that have contributed to the rise in 
NCDs include greater income; more mechanization and industrialization; improved access to food; urbanization; a globalization of unhealthy eating habits.

Compared on worldwide and regional levels, the chronic disease burden in Brazil can be further examined. In 2014, the total percentage of the population who died from an NCD in Brazil was 74\%; the total percentage of the population who died from an NCD in Latin America was 36\% in 2007 and worldwide was $68 \%$ in 2012 . These statistics indicate that the prevalence in Brazil exceeds both national and worldwide per- and alcohol consumption following behind, as shown in table 2. Currently, the highest trending risk factors in Brazil related to NCDs is overweight and obesity. In 2012, around one in seven Brazilians were classed as obese. Globally, Brazil is far behind countries such as the United States and Mexico, where around a third of people are obese, but the concern is the rapid rate of weight gain. With healthier foods becoming more expensive, lower income families have to opt to choose foods that may be higher in sugar and fat because they are the cheaper option. Collectively, the high prevalence of these risk factors are the triggers that

Table 2 Risk Factor Prevalence in Brazil, Data from World Health Organization

\begin{tabular}{|l|c|c|c|}
\hline & Male & Female & Total \\
\hline Obesity (2008) & $16.0 \%$ & $21.4 \%$ & $18.8 \%$ \\
\hline Tobacco Use (2011) & $22 \%$ & $13 \%$ & $17 \%$ \\
\hline Raised Blood Pressure (2008) & $36.8 \%$ & $25.3 \%$ & $30.8 \%$ \\
\hline Total alcohol per capita consumption, in liters (2010) & 13.6 & 4.2 & 8.7 \\
\hline
\end{tabular}

centages of deaths due to non-communicable diseases. If prevention measures are not properly implemented, and management of NCDs goes uncontrolled, the direct and indirect costs of NCDs will continue to pose important fiscal and public challenges for the country of Brazil.

\section{Risk Factors}

As mentioned earlier, the main risk factors that contribute to the development of NCD's include tobacco use, physical inactivity, unhealthy diet and the harmful use of alcohol. These factors are also main contributors for obesity and raised blood pressure rates, which greatly contribute to the development in NCDs. In Brazil, raised blood pressure in adults (18 or older) contributed as risk factor the total highest percentage, followed by obesity, tobacco use,

\section{Prevention Strategies}

Because of the extreme burden NCDs place on Brazil, several prevention strategies along with health promotion initiatives have been put in place that aim to combat the risk factors that lead to NCDs. In 2006, after discussion and agreement at federal, state, and municipal levels, a broad health promotion was approved. To further support local health promotion actives, the Ministry of Health contributed a great deal of funding to expand on the interventions put in place to combat risk factors and increase promotion of health. For example, to combat the highest trending risk factor of obesity, the country has launched a nationwide program to increase physical activity and improve diet, known as the Academia's da Saude. The Ministry of Health's goal is to expand Academia's da Saude of Brazil's promotion of physical 
activity to 4,000 municipalities by 2011 . By 2010, this physical activity network already included 469 projects. This is just one indicator that the promotion of a healthier lifestyle is a priority of Brazil's health system.

In 2006, to respond to the increasing prevalence of tobacco use, Brazil ratified the WHO Framework Convention on Tobacco Control. A free national hotline, whose number is placed within the cigarette pack warning, offers guidance on smoking cessation. Additionally, several new tobacco control bills have been approved at the national, state and municipal levels. In February, 2011, to respond to the increasing prevalence of those with raised blood pressure (hypertension), Brazil's Ministry of Health initiated the Health is Priceless Program. This program offers free medication to control and reduce the burden of hypertension, diabetes and asthma. The program distributes, free of charge, 11 medicines - six for hypertension and five for diabetes to control blood pressure and diabetes. As of February, 2014, the program, active in 4,119 cities through a network of 30,136 pharmacies, has provided services to 6.6 million diabetic and 16.4 million hypertensive patients. The three initiatives discussed within this section are an example of how Brazil has been taking action to combat their highest concerning risk factors for NCDs.

\section{Strategic Action Plan}

Looking towards the future of the noncommunicable disease burden in Brazil, the Unified Health System of Brazil (SUS) and the Ministry of Health set forth an NCD Action Plan 2011-2022. This action plan included a wide array of initiatives to respond to the ongoing epidemic of NCD's throughout Brazil. One aspect of the action plan included a National Health Survey administered in 2013. This survey was distributed for the purpose of producing data on national health status: risk behaviors; health services access; financing; etc. Data collection is very beneficial in the healthcare industry because it provides a deeper insight on the issue that has to be solved therefore makes it possible to produce the most appropriate solution to that specific issue, such as NCD prevention strategies. The strategic action plan also included targets to help reach their goals of reducing mortality and risk factors from NCDs. Some of the targets include reducing NCD premature death rate ( $<70$ years) by $2 \%$ a year; increasing fruit and vegetable consumption; reducing the prevalence of harmful alcohol consumption; reducing average salt intake; increasing the prevalence of physical activity.

Because the NCD burden is not a problem unique to Brazil and affects a majority of countries around the globe, The World Health Assembly endorsed the World Health Organization (WHO) Global Action Plan for the Prevention and Control of NCDs 20132020. This plan aims to reduce the number of premature deaths from NCDs by $25 \%$ by 2025 through nine voluntary global targets. The nine targets focus in part by addressing factors such as tobacco use; harmful use of alcohol; unhealthy diet; physical inactivities that increase people's risk of developing these diseases. The plan offers a menu of "best buy" or cost-effective, high-impact interventions for meeting the nine voluntary global targets such as banning all forms of tobacco and alcohol advertising; replacing trans fats with polyunsaturated fats; promoting and protecting breast feeding; preventing cervical cancer through screening. More than 190 countries, including Brazil, agreed in 2011 to adopt all nine voluntary targets in the WHO Global Action Plan 2013-2020. The adoption of the WHO voluntary targets were incorporated into their country's action plan. These initiatives show that Brazil is taking a proactive approach to reduce the burden of NCDs. 


\section{Conclusion}

In conclusion, it is clear to see how non-communicable diseases have become a major health priority in Brazil due to the large economic and social adverse effects they have throughout the communities within the country. On an individual level, NCDs impose financial, social, and health burdens. With obesity being the highest trending risk factor for NCDs due to unhealthy eating habits and a lack of physical activity, the initiate to promote a healthier lifestyle has to start at a young age. It is also important to stress that the most important way to reduce NCDs is to focus on lessening the risk factors associated with these diseases. In the long term, it is much less expensive for government to invest in prevention measures than to provide costly medical treatments to treat NCDs. In summary, Brazil has made significant progress in their efforts to reduce NCD by creating initiatives and having programs in place that have successfully reduced various risk factors such as tobacco use and raise blood pressure. Additionally, Brazil is active in its efforts both domestically and globally to address the threat of NCDs by striving to reach critical targets set out in their strategic action plan. Although progress has been made, comparing the NCD burden in Brazil on both a national and global level indicates that they have long roads ahead of them in order to reduce the mortality and morbidity rates associated with these diseases. With the continuous population growth and industrialization trends in Brazil, the NCD challenge will remain prevalent for years to come. So it is vital for healthcare sector and government in Brazil to remain proactive in their approach to reduce the NCD burden. In conclusion, Brazil has shown a great effort in reducing the NCD burden and in time, future data will allow the world to see the progress that has been made by the various initiatives set forth. 